\title{
SIFAT TRANSFORMASI LINIER ISOMETRI, OPERATOR SIMETRUS DAN TEOREMA SPEKTRAL
}

\author{
LATIFAH MUDHIANI, I MADE ARNAWA, NOVA NOLIZA BAKAR \\ Program Studi S1 Matematika, \\ Fakultas Matematika dan Ilmu Pengetahuan Alam, Universitas Andalas, \\ Kampus UNAND Limau Manis Padang, Indonesia. \\ email : latifahmudhiani7@gmail.com
}

Diterima 9 Maret 2019 Direvisi 7 April 2019 Dipublikasikan 7 Mei 2019

\begin{abstract}
Abstrak. Isometri adalah suatu transformasi linier dari ruang hasilkali dalam ke ruang hasilkali dalam yang memenuhi beberapa aksioma. Operator linier pada ruang hasilkali dalam $V$ ke $V$ yang memenuhi $\langle T(\mathbf{v}), \mathbf{w}\rangle=\langle\mathbf{v}, T(\mathbf{w})\rangle, \forall \mathbf{v}, \mathbf{w} \in V$, disebut operator self adjoint. Operator simetris adalah operator linier yang bernilai riil. Operator self adjoint merupakan konsep pendukung dari teorema spektral. Tulisan ini membahas sifat transformasi linier isometri, operator simetris, dan teorema spektral.
\end{abstract}

Kata Kunci: Isometri, Self Adjoint, Spektral

\section{Pendahuluan}

Transformasi linier merupakan suatu pemetaan yang memetakan suatu ruang vektor ke ruang vektor lainnya dan memenuhi beberapa aksioma terhadap operasi penjumlahan dan perkalian skalar. Ruang hasilkali dalam merupakan suatu ruang vektor yang dilengkapi dengan suatu hasilkali dalam.

Isometri merupakan suatu transformasi linier dari ruang hasilkali dalam ke ruang hasilkali dalam yang memenuhi beberapa aksioma. Transformasi linier dari ruang hasilkali dalam ke dirinya diri sendiri, disebut operator linier pada ruang hasilkali dalam. Operator linier pada ruang hasilkali dalam yang memenuhi aksioma tertentu, disebut operator self adjoint. Operator self adjoint yang bernilai riil, disebut operator simetris. Operator self adjoint merupakan suatu konsep pendukung untuk teorema spektral. Tulisan ini membahas sifat transformasi linier isometri, operator simetris, dan teorema spektral.

\section{Landasan Teori}

\subsection{Ruang Vektor}

Definisi 2.1. [3] Suatu ruang vektor $V$ atas lapangan $F$ adalah suatu himpunan $V$, dimana semua elemen-elemennya disebut vektor, yang memuat $\boldsymbol{O}$ dan dilengkapi dengan operasi penjumlahan dan perkalian dengan skalar, sehingga memenuhi syarat-syarat berikut: 
(a) Untuk semua $\boldsymbol{u}, \boldsymbol{v}, \boldsymbol{w} \in V$ berlaku:

(i) $\boldsymbol{u}+\boldsymbol{v} \in V$,

(ii) $\boldsymbol{O}+\boldsymbol{v}=\boldsymbol{v}$,

(iii) $\boldsymbol{u}+\boldsymbol{v}=\boldsymbol{v}+\boldsymbol{u}$,

(iv) $\boldsymbol{u}+(\boldsymbol{v}+\boldsymbol{w})=(\boldsymbol{u}+\boldsymbol{v})+\boldsymbol{w}$,

(b) Untuk semua $\boldsymbol{u}, \boldsymbol{v} \in V$ dan $r, s \in F$ berlaku:
(i) $r \boldsymbol{v} \in V$,
(ii) $1 \boldsymbol{v}=\boldsymbol{v}$
(iii) $0 \boldsymbol{v}=\boldsymbol{O}$,
(iv) $r(\boldsymbol{u}+\boldsymbol{v})=r \boldsymbol{u}+r \boldsymbol{v}$
(v) $(r+s) \boldsymbol{v}=r \boldsymbol{v}+s \boldsymbol{v}$,
(vi) $(r s) \boldsymbol{v}=r(s \boldsymbol{v})$.

Definisi 2.2. [3] Suatu subruang dari ruang vektor $V$ adalah suatu himpunan bagian $U \subseteq V$ yang mempunyai operasi yang sama dengan $V$ juga membentuk ruang vektor.

Lema 2.3. [3] Himpunan tak kosong $U \subseteq V$ dari ruang vektor $V$ adalah subruang dari $V$ jika dan hanya jika :

(a) Setiap $\boldsymbol{u}_{1}, \boldsymbol{u}_{2} \in U$ berlaku $\boldsymbol{u}_{1}+\boldsymbol{u}_{2} \in U$,

(b) Setiap $\boldsymbol{u} \in U$ dan $k \in F$ berlaku $k \boldsymbol{u} \in U$.

Definisi 2.4. [3] Himpunan vektor-vektor $\left\{\boldsymbol{v}_{1}, \boldsymbol{v}_{2}, \cdots, \boldsymbol{v}_{n}\right\}$ di ruang vektor $V$ disebut suatu basis untuk $V$, jika:

(a) $\boldsymbol{v}_{1}, \boldsymbol{v}_{2}, \cdots, \boldsymbol{v}_{n}$ bebas linier,

(b) $\operatorname{span}\left\{\boldsymbol{v}_{1}, \boldsymbol{v}_{2}, \cdots, \boldsymbol{v}_{n}\right\}=V$.

\subsection{Transformasi Linier}

Definisi 2.5. [3] Misalkan $V$ dan $W$ ruang vektor atas lapangan riil F. Suatu transformasi linier dari $V$ ke $W$ adalah suatu fungsi $T: V \longrightarrow W$ yang memenuhi:

(a) Jika $\boldsymbol{u}, \boldsymbol{v} \in V$, maka $T(\boldsymbol{u}+\boldsymbol{v})=T(\boldsymbol{u})+T(\boldsymbol{v})$,

(b) Jika $\boldsymbol{v} \in V$ dan $k \in F$, maka $T(k \boldsymbol{v})=k T(\boldsymbol{v})$.

Teorema 2.6. [3] Asumsikan $V$ dan $W$ adalah ruang vektor. Misalkan $\left\{\boldsymbol{v}_{1}, \boldsymbol{v}_{2}, \cdots, \boldsymbol{v}_{n}\right\}$ basis dari $V$, dan $\boldsymbol{w}_{1}, \boldsymbol{w}_{2}, \cdots, \boldsymbol{w}_{n} \in W$. Maka terdapat tunggal $T: V \longrightarrow W$ yang memenuhi

$$
T\left(\boldsymbol{v}_{1}\right)=\boldsymbol{w}_{1}, T\left(\boldsymbol{v}_{2}\right)=\boldsymbol{w}_{2}, \cdots, T\left(\boldsymbol{v}_{n}\right)=\boldsymbol{w}_{n}
$$

Definisi 2.7. [3] Misalkan A matriks $m \times n$ maka perkalian A dari kiri dapat dipandang sehingga suatu fungsi dari $F^{n}$ ke $F^{m}$. Fungsi ini dinyatakan sebagai $T_{A}: F^{n} \longrightarrow F^{m}$. Dengan kata lain

$$
T_{A}(\boldsymbol{v})=A \boldsymbol{v} \quad, \forall \boldsymbol{v} \in F^{n}
$$

Definisi 2.8. [3] 
Sifat Transformasi Linier Isometri, Operator Simetris, dan Teorema Spektral 173

(a) Transformasi linier $T: V \longrightarrow W$ disebut satu-satu (injektif) jika setiap $\boldsymbol{u}, \boldsymbol{v} \in$ $V$ dengan $T(\boldsymbol{u})=T(\boldsymbol{v})$ maka $\boldsymbol{u}=\boldsymbol{v}$,

(b) Transformasi linier $T$ disebut pada (surjektif) jika im $(T)=W$,

(c) Transformasi linier $T$ disebut isomorfisma jika $T$ satu-satu dan pada (bijektif),

(d) Jika terdapat suatu isomorfisma $T: V \longrightarrow W$, maka dikatakan ruang vektor $V$ dan $W$ adalah isomorfik,

(e) Suatu isomorfisma $T: V \longrightarrow W$ disebut operator linier taksingular.

Teorema 2.9. [3] Misalkan $V$ dan $W$ ruang vektor yang berdimensi hingga dan $T: V \longrightarrow W$ transformasi linier. Maka

(a) T satu-satu jika dan hanya jika $\operatorname{ker}(T)=\{\boldsymbol{O}\}$,

(b) $T$ satu-satu jika dan hanya jika $\boldsymbol{v}_{1}, \boldsymbol{v}_{2}, \cdots, \boldsymbol{v}_{n}$ bebas linier di $V$, $\left\{T\left(\boldsymbol{v}_{1}\right), T\left(\boldsymbol{v}_{2}\right), \cdots, T\left(\boldsymbol{v}_{n}\right)\right\} \in W$ bebas linier di $W$,

(c) $T$ pada jika dan hanya jika $r k(T)=\operatorname{dim}(W)$,

(d) $T$ isomorfisma jika dan hanya jika $\left\{\boldsymbol{v}_{1}, \boldsymbol{v}_{2}, \cdots, \boldsymbol{v}_{n}\right\}$ basis dari $V$, $\left\{T\left(\boldsymbol{v}_{1}\right), T\left(\boldsymbol{v}_{2}\right), \cdots, T\left(\boldsymbol{v}_{n}\right)\right\}$ basis dari $W$,

(e) Jika $T$ isomorfisma, maka $\operatorname{dim}(V)=\operatorname{dim}(W)$,

(f) Jika $T$ isomorfisma, maka $T^{-1}$ ada sebagai fungsi dan $T^{-1}: W \longrightarrow V$ juga suatu isomorfisma.

Definisi 2.10. [3] Misalkan $T: V \longrightarrow W$ suatu transformasi linier, $\mathfrak{B}=\left\{\boldsymbol{v}_{1}, \boldsymbol{v}_{2}, \cdots, \boldsymbol{v}_{n}\right\}$ basis terurut untuk $V, \mathfrak{C}=\left\{\boldsymbol{w}_{1}, \boldsymbol{w}_{2}, \cdots, \boldsymbol{w}_{m}\right\}$ basis terurut untuk $W$, didefinisikan matriks $[T]_{\mathfrak{B}, \mathfrak{C}}$ yang berukuran $m \times n$ sebagai $[T]_{\mathfrak{B}, \mathfrak{C}}=\left(a_{i j}\right)$ dimana untuk setiap $j$ skalar $a_{i j}$ ditentukan secara tunggal oleh persamaan

$$
T\left(\boldsymbol{v}_{j}\right)=a_{1 j} \boldsymbol{w}_{1}+a_{2 j} \boldsymbol{w}_{2}+\cdots+a_{m j} \boldsymbol{w}_{m}
$$

Dengan kata lain $[T]_{\mathfrak{B}, \mathfrak{C}}$ adalah matriks dimana kolom-kolomnya adalah koordinat $T\left(\boldsymbol{v}_{j}\right)$ yang berkaitan dengan basis terurut $\mathfrak{C}$ untuk $W$.

Definisi 2.11. [3]

(a) Misalkan $T: V \longrightarrow V$ adalah operator linier. Jika $\boldsymbol{v} \in V$ adalah vektor taknol dan terdapat skalar $k$ sedemikian sehingga $T(\boldsymbol{v})=k \boldsymbol{v}$, maka dikatakan $\boldsymbol{v}$ vektor eigen dari T. Skalar $k$ disebut nilai eigen dari $T$ yang terkait dengan vektor eigen $\boldsymbol{v}$

(b) Jika A suatu matriks $n \times n$, maka nilai eigen dari A diartikan sebagai nilai eigen $\operatorname{dari} T_{A}$.

\subsection{Ruang Hasilkali Dalam}

Definisi 2.12. [3] Misalkan $V$ suatu ruang vektor riil. Suatu hasilkali dalam riil pada $V$ adalah suatu fungsi bernilai riil pada $V \times V$, biasa dinyatakan sebagai $\langle$, yang memenuhi:

(a) $\langle\boldsymbol{u}, \boldsymbol{v}\rangle=\langle\boldsymbol{v}, \boldsymbol{u}\rangle$ untuk setiap $\boldsymbol{u}, \boldsymbol{v} \in V$,

(b) $\langle k \boldsymbol{u}, \boldsymbol{v}\rangle=k\langle\boldsymbol{u}, \boldsymbol{v}\rangle$ untuk setiap $k \in \mathbb{R}$ dan $\boldsymbol{u}, \boldsymbol{v} \in V$,

(c) $\left\langle\boldsymbol{v}_{1}+\boldsymbol{v}_{2}, \boldsymbol{u}\right\rangle=\left\langle\boldsymbol{v}_{1}, \boldsymbol{u}\right\rangle+\left\langle\boldsymbol{v}_{2}, \boldsymbol{u}\right\rangle$ untuk setiap $\boldsymbol{v}_{1}, \boldsymbol{v}_{2}, \boldsymbol{u} \in V$, 
174 Latifah Mudhiani dkk

(d) $\langle\boldsymbol{v}, \boldsymbol{v}\rangle \geq 0$ untuk setiap $\boldsymbol{v} \in V$, dan $\langle\boldsymbol{v}, \boldsymbol{v}\rangle=0$ jika dan hanya jika $\boldsymbol{v}=\boldsymbol{O}$.

Definisi 2.13. [3] Misalkan V suatu ruang vektor kompleks. Suatu hasilkali dalam kompleks pada $V$ adalah suatu fungsi bernilai kompleks pada $V \times V$, biasa dinyatakan sebagai $\langle$,$\rangle yang memenuhi:$

(a) $\langle\boldsymbol{u}, \boldsymbol{v}\rangle=\overline{\langle\boldsymbol{v}, \boldsymbol{u}\rangle}$ untuk setiap $\boldsymbol{u}, \boldsymbol{v} \in V$,

(b) $\langle k \boldsymbol{u}, \boldsymbol{v}\rangle=k\langle\boldsymbol{u}, \boldsymbol{v}\rangle$ untuk setiap $k \in \mathbb{C}$ dan $\boldsymbol{u}, \boldsymbol{v} \in V$,

(c) $\left\langle\boldsymbol{v}_{1}+\boldsymbol{v}_{2}, \boldsymbol{u}\right\rangle=\left\langle\boldsymbol{v}_{1}, \boldsymbol{u}\right\rangle+\left\langle\boldsymbol{v}_{2}, \boldsymbol{u}\right\rangle$ untuk setiap $\boldsymbol{v}_{1}, \boldsymbol{v}_{2}, \boldsymbol{u} \in V$,

(d) $\langle\boldsymbol{v}, \boldsymbol{v}\rangle \in \mathbb{R}$ dan $\langle\boldsymbol{v}, \boldsymbol{v}\rangle \geq 0$ untuk setiap $\boldsymbol{v} \in V$, dan $\langle\boldsymbol{v}, \boldsymbol{v}\rangle=0$ jika dan hanya jika $\boldsymbol{v}=\boldsymbol{O}$

Definisi 2.14. [3]

(a) Misalkan $V$ suatu ruang vektor atas $\mathbb{R}, V$ disebut ruang hasilkali dalam riil jika di $V$ dapat didefinisikan suatu hasilkali dalam riil,

(b) Misalkan $V$ suatu ruang vektor atas $\mathbb{C}, V$ disebut ruang hasilkali dalam kompleks jika di $V$ dapat didefinisikan suatu hasilkali dalam kompleks.

Definisi 2.15. [3] Jika $V$ adalah ruang vektor, $\boldsymbol{v} \in V$, dan $\langle$,$\rangle adalah hasilkali$ dalam pada $V$ maka didefinisikan $\|\boldsymbol{v}\|=\langle\boldsymbol{v}, \boldsymbol{v}\rangle^{1 / 2}$. Fungsi ini \|\|$: V \longrightarrow V$ disebut norm yang terkait dengan hasilkali dalam.

Definisi 2.16. [5]

(a) Misalkan $\boldsymbol{u}=\left(u_{1}, u_{2}, \cdots, u_{n}\right)$ dan $\boldsymbol{v}=\left(v_{1}, v_{2}, \cdots, v_{n}\right)$ adalah vektor kolom, dengan $\boldsymbol{u}, \boldsymbol{v} \in R^{n}$, hasilkali dalam dapat didefinisikan

$$
\langle\boldsymbol{u}, \boldsymbol{v}\rangle=\boldsymbol{u}^{t} \boldsymbol{v},
$$

(b) Misalkan $\boldsymbol{u}=\left(u_{1}, u_{2}, \cdots, u_{n}\right)$ dan $\boldsymbol{v}=\left(v_{1}, v_{2}, \cdots, v_{n}\right)$ adalah vektor kolom, dengan $\boldsymbol{u}, \boldsymbol{v} \in C^{n}$, hasilkali dalam dapat didefinisikan

$$
\langle\boldsymbol{u}, \boldsymbol{v}\rangle=\boldsymbol{u}^{t} \overline{\boldsymbol{v}}
$$

Definisi 2.17. [2] Misalkan $V$ suatu ruang hasilkali dalam jiika $\boldsymbol{u}, \boldsymbol{v} \in V$, maka $\boldsymbol{u}$ disebut orthogonal dengan $\boldsymbol{v}$ jika $\langle\boldsymbol{u}, \boldsymbol{v}\rangle=0$.

Definisi 2.18. [3] Misalkan $\langle$,$\rangle suatu hasilkali dalam pada ruang vektor V$. Basis $\left\{\boldsymbol{u}_{1}, \boldsymbol{u}_{2}, \cdots, \boldsymbol{u}_{n}\right\}$ adalah basis orthogonal jika $u_{i}$ saling orthogonal, yakni $\left\langle\boldsymbol{u}_{i}, \boldsymbol{u}_{j}\right\rangle=$ 0 dengan $i \neq j$. Selain itu, jika $\left\|u_{i}\right\|=1$ disebut basis orthonormal.

Teorema 2.19. [3] Misalkan $\boldsymbol{u}_{1}, \boldsymbol{u}_{2}, \cdots, \boldsymbol{u}_{n}$ basis orthonormal dari ruang vektor $V$ dengan hasilkali dalam $\langle$,$\rangle , maka untuk setiap \boldsymbol{v} \in V$,

$$
\boldsymbol{v}=a_{1} \boldsymbol{u}_{1}+a_{2} \boldsymbol{u}_{2}+\cdots+a_{n} \boldsymbol{u}_{n}
$$

dimana $a_{i}=\left\langle\boldsymbol{v}, \boldsymbol{u}_{i}\right\rangle$.

Definisi 2.20. [3] Jika $\langle$,$\rangle adalah suatu hasilkali dalam pada V$ dan $\boldsymbol{u}, \boldsymbol{v} \neq \boldsymbol{O} \in V$, didefinisikan proyeksi orthogonal dari $\boldsymbol{u}$ pada $\boldsymbol{v}$ sebagai

$$
\operatorname{proj}_{\boldsymbol{v}}(\boldsymbol{u})=\frac{\langle\boldsymbol{u}, \boldsymbol{v}\rangle}{\langle\boldsymbol{v}, \boldsymbol{v}\rangle} \boldsymbol{v}
$$


Teorema 2.21. [1] Setiap ruang hasilkali dalam taknol berdimensi hingga memiliki sebuah basis orthonormal.

Definisi 2.22. [3] Sebuah matriks riil $U_{n \times n}$ disebut orthogonal jika $U U^{t}=I_{n}$; yakni, $U$ dapat dibalik dan $U^{-1}=U^{t}$. Jika matriks kompleks $A$ adalah matriks kompleks $n \times n$, maka $A^{*}=\overline{(A)}^{t}$. Sebuah matriks kompleks $U_{n \times n}$ disebut uniter jika $U U^{*}=I_{n}$; yakni, $U$ dapat dibalik dan $U^{-1}=U^{*}$.

Lema 2.23. [3]

(a) Sebuah matriks U orthogonal jika dan hanya jika baris (kolom) orthonormal dengan hasilkali titik,

(b) Sebuah matriks kompleks U uniter jika dan hanya jika baris (kolom) orthonormal pada ruang uniter $\mathbb{C}^{n}$.

\subsection{Proyeksi Orthogonal dan Jumlah Langsung}

Definisi 2.24. [3] Misalkan $V$ suatu ruang hasilkali dalam dan $S \subseteq V$. Komplement orthogonal $S$ di $V$, ditulis $S^{\perp}$, didefinisikan sebagai

$$
S^{\perp}=\{\boldsymbol{v} \in V \mid\langle\boldsymbol{v}, \boldsymbol{w}\rangle=0 \text { untuk setiap } \boldsymbol{w} \in S\}
$$

Jika $W$ subruang berdimensi hingga dari $V$, proyeksi orthogonal $\operatorname{Proj}_{W}: V \longrightarrow V$ adalah operator linier dengan

$$
\begin{gathered}
\operatorname{proj}_{W}(\boldsymbol{w})=\boldsymbol{w} \text { untuk } \boldsymbol{w} \in W \text { dan } \\
\operatorname{proj}_{W}(\boldsymbol{v})=\boldsymbol{O} \text { untuk } \boldsymbol{v} \in W^{\perp}
\end{gathered}
$$

Teorema 2.25. [3] Misalkan $\langle$,$\rangle adalah hasilkali dalam pada V$ dan $W$ subruang berdimensi hingga dari $V$. Maka terdapat proyeksi orthogonal

$\operatorname{Proj}_{W}: V \longrightarrow V$. Misalkan $\left\{\boldsymbol{u}_{1}, \boldsymbol{u}_{2}, \cdots, \boldsymbol{u}_{s}\right\}$ suatu basis orthonormal dari $W$. Proyeksi orthogonal Projw dapat dicari dengan rumus

$$
\operatorname{Proj}_{W}(\boldsymbol{v})=\left\langle\boldsymbol{v}, \boldsymbol{u}_{1}\right\rangle \boldsymbol{u}_{1}+\left\langle\boldsymbol{v}, \boldsymbol{u}_{2}\right\rangle \boldsymbol{u}_{1}+\cdots+\left\langle\boldsymbol{v}, \boldsymbol{u}_{s}\right\rangle \boldsymbol{u}_{s} .
$$

Definisi 2.26. [3] Misalkan $W_{1}$ dan $W_{2}$ subruang dari ruang vektor $V$. Ruang vektor $V=W_{1} \oplus W_{2} j i k a$

(a) $W_{1} \cap W_{2}=\{\boldsymbol{O}\}$,

(b) $V=W_{1}+W_{2}$, yakni untuk setiap $\boldsymbol{v} \in V$, dapat ditulis menjadi

$\boldsymbol{v}=\boldsymbol{w}_{1}+\boldsymbol{w}_{2}$, untuk $\boldsymbol{w}_{1} \in W_{1}$ dan $\boldsymbol{w}_{2} \in W_{2}$,

$V$ disebut jumlah langsung dari $W_{1}$ dan $W_{2}$.

Akibat 2.27. [3] Misalkan $V$ ruang hasilkali dalam berdimensi hingga dan $W$ subruang dari $V$.

(a) $\operatorname{Jika} \operatorname{dim}(V)=n$ dan $\operatorname{dim}(W)=s$, maka $\operatorname{dim}\left(W^{\perp}\right)=n-s$,

(b) Untuk setiap $\boldsymbol{v} \in V$, terdapat $\boldsymbol{w}_{1} \in W$ dan $\boldsymbol{w}_{2} \in W^{\perp}$, sehingga $\boldsymbol{v}=\boldsymbol{w}_{1}+\boldsymbol{w}_{2}$. Di kasus ini $\boldsymbol{w}_{1}=\operatorname{Proj}_{W}$ dan $\boldsymbol{w}_{2}=\operatorname{Proj}_{W^{\perp}}$, yang menunjukkan $\operatorname{Proj}_{W}+\operatorname{Proj}_{W \perp}=I$. 


\section{Pembahasan}

Definisi 3.1. [3] Misalkan $\langle,\rangle_{1}: V_{1} \times V_{1} \longrightarrow F$ adalah hasilkali dalam di $V_{1}$, dan $\langle,\rangle_{2}: V_{2} \times V_{2} \longrightarrow F$ adalah hasilkali dalam di $V_{2}$

(a) Transformasi linier $T$ dari ruang hasilkali dalam $V_{1}$ ke ruang hasilkali dalam $V_{2}$ disebut isometri, jika

(i) $T$ isomorfisma,

(ii) $\langle\boldsymbol{v}, \boldsymbol{w}\rangle_{1}=\langle T(\boldsymbol{v}), T(\boldsymbol{w})\rangle_{2}$ untuk setiap $\boldsymbol{v}, \boldsymbol{w} \in V_{1}$,

(b) $V_{1}$ dan $V_{2}$ disebut isometrik jika ada pemetaan isometri dari $V_{1}$ ke $V_{2}$.

Definisi 3.2. [3]

(a) Misalkan $T: V \longrightarrow V$ operator linier pada ruang hasilkali dalam $V$. $T$ disebut self adjoint jika setiap $\boldsymbol{v}, \boldsymbol{w} \in V,\langle T(\boldsymbol{v}), \boldsymbol{w}\rangle=\langle\boldsymbol{v}, T(\boldsymbol{w})\rangle$.

(b) Misalkan $T$ suatu operator self adjoint dari $V$ ke $V$, jika $V$ adalah suatu ruang vektor rill, maka $T$ disebut operator simetris.

(c) Misalkan $T$ suatu operator self adjoint dari $V$ ke $V$, jika $V$ adalah suatu ruang vektor kompleks, maka $T$ disebut operator hermitian.

(d) Sebuah matriks riil $A$ disebut self adjoint atau simetris, jika $A^{t}=A$.

(e) Sebuah matriks kompleks $A$ disebut self adjoint atau hermitian, jika $A^{*}=A$.

\subsection{Sifat Transformasi Linier Isometri dan Operator Simetris}

Lema 3.3. [3] Misalkan $V$ dan $W$ ruang hasilkali dalam berdimensi hingga, $T$ : $V \longrightarrow W$ transformasi linier. Pernyataan berikut ekuivalen

(a) T isometri,

(b) Jika $\left\{\boldsymbol{u}_{1}, \boldsymbol{u}_{2}, \cdots, \boldsymbol{u}_{n}\right\}$ basis orthonormal dari $V$, maka $\left\{T\left(\boldsymbol{u}_{1}\right), T\left(\boldsymbol{u}_{2}\right)\right.$, $\left.\cdots, T\left(\boldsymbol{u}_{n}\right)\right\}$ basis orthonormal dari $W$.

Lema 3.4. [3]

(a) Misalkan $T$ operator linier di $\mathbb{R}^{n}, T: \mathbb{R}^{n} \longrightarrow \mathbb{R}^{n}$ isometri (dengan hasilkali titik) jika dan hanya jika matriks standar $[T]$ adalah orthogonal.

(b) Misalkan T operator linier di $\mathbb{C}^{n}$, operator linier $T: \mathbb{C}^{n} \longrightarrow \mathbb{C}^{n}$ isometri jika dan hanya jika matriks standar $[T]$ uniter.

(c) Dua ruang hasilkali dalam riil berdimensi hingga adalah isometrik jika dan hanya jika kedua ruang hasilkali dalam riil berdimensi hingga mempunyai dimensi yang sama.

(d) Dua ruang hasilkali dalam kompleks berdimensi hingga adalah isometrik jika dan hanya jika kedua ruang hasilkali dalam kompleks berdimensi hingga mempunyai dimensi yang sama.

Lema 3.5. [3]

(a) Jika $A \in M_{n \times n}(\mathbb{C}) \operatorname{maka} \forall \boldsymbol{v}, \boldsymbol{w} \in \mathbb{C}^{n}$

$$
\langle A \boldsymbol{v}, \boldsymbol{w}\rangle=\left\langle\boldsymbol{v}, A^{*} \boldsymbol{w}\right\rangle
$$


Sifat Transformasi Linier Isometri, Operator Simetris, dan Teorema Spektral 177

(b) Misalkan $F=\mathbb{R}$ atau $\mathbb{C}$ jika $T: F^{n} \longrightarrow F^{n}$ operator linier, maka $T$ adalah self adjoint jika dan hanya jika $[T]$ adalah self adjoint.

Teorema 3.6. [3] Setiap operator self adjoint $T: V \longrightarrow V$ pada ruang hasilkali dalam $V$ berdimensi hingga mempunyai nilai eigen riil.

Bukti. Misalkan setiap operator self adjoint $T: V \longrightarrow V$ pada ruang hasilkali dalam $V$ berdimensi hingga, akan ditunjukkan mempunyai nilai eigen riil.

Perhatikan bahwa

$$
\begin{aligned}
\langle\mathbf{v}, T(\mathbf{v})\rangle & =\langle\mathbf{v}, \lambda \mathbf{v}\rangle & & \text { (Definisi 2.11) } \\
& =\bar{\lambda}\langle\mathbf{v}, \mathbf{v}\rangle & & \text { (Definisi 2.13) } \\
& =\bar{\lambda}\|\mathbf{v}\|^{2} & & \\
\langle T(\mathbf{v}), \mathbf{v}\rangle & =\langle\lambda \mathbf{v}, \mathbf{v}\rangle & & \text { (Definisi 2.11) } \\
& =\lambda\langle\mathbf{v}, \mathbf{v}\rangle & & \text { (Definisi 2.12) } \\
& =\lambda\|\mathbf{v}\|^{2} & &
\end{aligned}
$$

Dari Persamaan (3.2) dan (3.3) diperoleh $\bar{\lambda}=\lambda$. Ini berarti $\lambda \in \mathbb{R}$.

Teorema 3.7. [3] $T: V \longrightarrow V$ adalah operator self adjoint, dimana $V$ adalah ruang hasilkali dalam berdimensi hingga. Maka $V$ mempunyai basis orthonormal dari vektor eigen $T$.

\subsection{Teorema Spektral}

Teorema 3.8. [3] $T: V \longrightarrow V$ adalah operator self adjoint dari ruang hasilkali dalam $V$ berdimensi hingga. Maka ada subruang yang saling orthogonal $W_{1}, W_{2}, \cdots, W_{s}$ dari $V$, dan dengan bilangan riil $r_{1}, r_{2}, \cdots, r_{s}$ yang berbeda, sehingga

$$
\begin{aligned}
T & =r_{1} \operatorname{Proj}_{W_{1}}+r_{2} \operatorname{Proj}_{W_{2}}+\cdots+r_{s} \operatorname{Proj}_{W_{s}} \\
\text { dan } I_{v} & =\operatorname{Proj}_{W_{1}}+\operatorname{Proj}_{W_{2}}+\cdots+\operatorname{Proj}_{W_{s}}
\end{aligned}
$$

dimana $I_{v}: V \longrightarrow V$ operator identitas. $T$ disebut resolusi spektral dari $T$.

\section{Kesimpulan}

(1) Misalkan $V$ dan $W$ ruang hasilkali dalam berdimensi hingga, $T: V \longrightarrow W$ transformasi linier. Pernyataan berikut ekuivalen

(a) $T$ isometri,

(b) Jika $\left\{\mathbf{u}_{1}, \mathbf{u}_{2}, \cdots, \mathbf{u}_{n}\right\}$ basis orthonormal dari $V$, maka $\left\{T\left(\mathbf{u}_{1}\right), T\left(\mathbf{u}_{2}\right)\right.$, $\left.\cdots, T\left(\mathbf{u}_{n}\right)\right\}$ basis orthonormal dari $W$.

(2)(a) Misalkan $\mathrm{T}$ operator linier di $\mathbb{R}^{n}, T: \mathbb{R}^{n} \longrightarrow \mathbb{R}^{n}$ isometri (dengan hasilkali titik) jika dan hanya jika matriks standar $[T]$ adalah orthogonal.

(b) Misalkan $\mathrm{T}$ operator linier di $\mathbb{C}^{n}$, operator linier $T: \mathbb{C}^{n} \longrightarrow \mathbb{C}^{n}$ isometri jika dan hanya jika matriks standar $[T]$ uniter. 
(c) Dua ruang hasilkali dalam riil berdimensi hingga adalah isometrik jika dan hanya jika kedua ruang hasilkali dalam riil berdimensi hingga mempunyai dimensi yang sama.

(d) Dua ruang hasilkali dalam kompleks berdimensi hingga adalah isometrik jika dan hanya jika kedua ruang hasilkali dalam kompleks berdimensi hingga mempunyai dimensi yang sama.

(3)(a) Jika $A \in M_{n \times n}(\mathbb{C})$ maka $\forall \mathbf{v}, \mathbf{w} \in \mathbb{C}^{n}$

$$
\langle A \mathbf{v}, \mathbf{w}\rangle=\left\langle\mathbf{v}, A^{*} \mathbf{w}\right\rangle
$$

(b) Misalkan $F=\mathbb{R}$ atau $\mathbb{C}$ jika $T: F^{n} \longrightarrow F^{n}$ operator linier, maka $T$ adalah self adjoint jika dan hanya jika $[T]$ adalah self adjoint.

(4) Setiap operator self adjoint $T: V \longrightarrow V$ pada ruang hasilkali dalam $V$ berdimensi hingga mempunyai nilai eigen riil.

(5) $T: V \longrightarrow V$ adalah operator self adjoint, dimana $V$ adalah ruang hasilkali dalam berdimensi hingga. Maka $V$ mempunyai basis orthonormal dari vektor eigen $T$.

(6) $T: V \longrightarrow V$ adalah operator self adjoint dari ruang hasilkali dalam $V$ berdimensi hingga. Maka ada subruang yang saling orthogonal $W_{1}, W_{2}, \cdots, W_{s}$ dari $V$, dan dengan bilangan riil $r_{1}, r_{2}, \cdots, r_{s}$ yang berbeda,sehingga

$$
\begin{aligned}
T & =r_{1} \operatorname{Proj}_{W_{1}}+r_{2} \operatorname{Proj}_{W_{2}}+\cdots+r_{s} \operatorname{Proj}_{W_{s}} \\
\text { dan } I_{v} & =\operatorname{Proj}_{W_{1}}+\operatorname{Proj}_{W_{2}}+\cdots+\operatorname{Proj}_{W_{s}}
\end{aligned}
$$

dimana $I_{v}: V \longrightarrow V$ operator identitas. $T$ disebut resolusi spektral dari $T$.

\section{Ucapan Terima kasih}

Penulis mengucapkan terima kasih kepada Ibu Yanita, Ibu Monika Rianti Helmi, dan Ibu Lyra Yulianti yang telah memberikan masukan dan saran sehingga makalah ini dapat diselesaikan dengan baik.

\section{Daftar Pustaka}

[1] Anton, H dan C. Rorres. 2004. Aljabar Linier Elementer. Edisi Kedelapan. Erlangga, Jakarta

[2] Herstein, I.N. 2000. Topics in Algebra. Second Edition. John Wiley \& Sons, Singapore

[3] Jacob, B. 1990. Linear Algebra. W. H. Freeman and Company, New York

[4] Lang, S. 1987. Linier Algebra. Third Edition. Springer-Verlag New York,Inc. Amerika Serikat

[5] Lipschutz, S dan M.L. Lipson. 2009. Schaum's Outlines, Linear Algebra. Edisi Empat. McGraw-Hill Companies, United States 